\title{
DWDM over FSO under the effect of different atmospheric attenuations
}

\author{
Heyam Maraha ${ }^{1}$, Kameran Ali Ameen², Ozlam Abdulhakeem Mahmood ${ }^{3}$, Aras Al-dawoodi ${ }^{4}$ \\ ${ }^{1,3}$ Network Department, College of Computer Science and Information Technology, Kirkuk University, Iraq \\ ${ }^{2,4}$ Computer Science Department, College of Computer Science and Information Technology, Kirkuk University, Iraq
}

\begin{tabular}{l} 
Article Info \\
\hline Article history: \\
Received Jul 30, 2019 \\
Revised Oct 31, 2019 \\
Accepted Nov 14, 2019 \\
\hline
\end{tabular}

Keywords:

Atmospheric attenuations Different weather condition DWDM

Electrical linear equalizer FSO

Steganography

\begin{abstract}
In optical networks, Dense wavelength division multiplexing (DWDM) has been considered as a promising technique to meet the increasing bandwidth demands. It has been used to increase the capacity of long-haul optical transport systems such as free-space optics (FSO) and optical fiber. However, by applying DWDM over the FSO link, different challenges affect the performance system such as clear air, haze and rain atmospheric attenuations. This paper investigates eleven-channel DWDM over FSO based on the electrical linear equalizer that will improve the performance results at the receiver. The channels operate over $(1561.42 \mathrm{~nm}, 1559.79 \mathrm{~nm}, 1558.17$ $\mathrm{nm}, 1558.55 \mathrm{~nm}, 1554.94 \mathrm{~nm}, 1553.33 \mathrm{~nm}, 1551.72 \mathrm{~nm}, 1550.12 \mathrm{~nm}$, $1548.51 \mathrm{~nm}, 1546.92$ and $1545.32 \mathrm{~nm}$ ) wavelengths that have separated based on the traditional International Telecommunication Union (ITU) grid. In the experiments, the system transmits $110 \mathrm{Gbit} / \mathrm{s}$ for FSO distances 9500 $\mathrm{m}, 3000 \mathrm{~m}$, and $2500 \mathrm{~m}$ in superbly clear air, haze, and heavy haze atmospheric attenuations, respectively. Over different atmospheric attenuations, we evaluated our system performance using BER, eye diagram and the quality factor (Q-Factor).
\end{abstract}

Copyright @ 2020 Institute of Advanced Engineering and Science. All rights reserved.

Corresponding Author:

Aras Al-dawoodi,

Computer Science Department,

College of Computer Science and Information Technology,

Kirkuk, Iraq.

Email: aras.ghazi86@uokirkuk.edu.iq

\section{INTRODUCTION}

In the last decade, a rapid increase has been seen in traffic demand due to the invention of different needs to large data downloads, such as gaming systems (Play station, Xbox, etc.). These systems require onboard storage to enable players to download software and games rather than buying on DVDs. It is expected that by 2022, the large files of graphically intense games are expected to take 4 percent of all IPs [1]. Additionally, during peak usage periods, these downloads tend to occur with gaming downloads getting up to 8 percent of heavy hour traffic. Besides, by 2022 also, the rapid growth of gaming traffic is anticipated to increase, and the gaming will be crucial in limiting the likelihood that video traffic will exceed the projected 82 percent [2]. However, different multiplexing has been developed to overcome as follows [3-9]:

a. Wavelength Division Multiplexing (WDM).

b. Space Division Multiplexing (SDM).

c. Orthogonal Frequency-Division Multiple Access (OFDMA).

d. Optical Code Division Multiple Access (OCDMA).

These multiplexing methods can be worked on a different medium like optical wireless and fiber optics. FSO plays an important medium to transfer data over different atmospheric attenuations $[3-4,6,8,10]$. 
Recently, the FSO communication system has been implemented as an efficient solution to tackle the fall of internet service providers to the higher data rate demands. In FSO systems, by using the air, the light signal transmits to the destination as a transmission medium. It is well known that FSO systems have several pros, such as easy and fast deployment, full-duplex transmission, high transmission security, bandwidth, scalability, high bit rates, license-free operation, etc. [11-14]. The wireless telecommunication system of FSO utilizes a transmission medium of free space to transmit optical data at high bit rates.

This study applies DWDM for FSO that operate over (1561.42 nm, $1559.79 \mathrm{~nm}, 1558.17 \mathrm{~nm}$, $1558.55 \mathrm{~nm}, 1554.94 \mathrm{~nm}, 1553.33 \mathrm{~nm}, 1551.72 \mathrm{~nm}, 1550.12 \mathrm{~nm}, 1548.51 \mathrm{~nm}, 1546.92$, and $1545.32 \mathrm{~nm})$ wavelength to improve the capacity. In the optical networks, DWDM technology became the main choice for fulfilling the rapidly increasing bandwidth demands [15]. Furthermore, it has been implemented to increase the capacity of long-haul optical transport systems [16]. Researches have paid more attention to move this promising technology into the broadband access networks that serve business and residential clients [17]. Moreover, on a single optical, DWDM enables multiple signals to be transmitted simultaneously at several wavelengths and implement to increase bandwidth over the optical communication networks [18].

\section{LITERATURE REVIEW}

Different researchers applied DWDM over different transmission mediums such as (SMF, OWC, and FSO) based on different distances and capacities. In 2014, the author in [19] applied a 64 channel DWDM system based on RZ modulation and $150 \mathrm{MHz}$ channel spacing that gives optimal BER and Qfactors after $60 \mathrm{Km}$ SMF links. In 2016, the author in [20], successfully demonstrated $40 \mathrm{~GB} / \mathrm{s}$ DWDM over $4000 \mathrm{~m}$ FSO link system. The performance results investigated on the designed DWDM over FSO and designed using RZ modulation with a number of parameters: 1) FSO transmitter and receiver diameter; 2) signal power; 3) beam divergence; and 4) different distances and attenuation. In 2017, under clear weather conditions, $80 \mathrm{~GB} / \mathrm{s}$ DWDM system had been simulated with the spacing of $100 \mathrm{GHz}$ frequency under turbulence incorporated over $38500 \mathrm{~km}$ optical wireless communication (OWC) [21]. The work showed that the Q-factor increased (decreased) with the increase in transmitted optical power (the increase of turbulence effects).

Recently, the authors in [22] designed and simulated a 32-channel DWDM-FSO link system over 40 Gbps. The system was implemented in moderate attenuation and turbulence-model (Gamma-Gamma) link. The findings were very encouraging as compensation schemes pre/post/symmetric) improve the effective coverage area. Furthermore, in the case of uncompensated, it was restricted to a maximum of $2 \mathrm{~km}$. However, by applying DWDM over FSO link different atmospheric attenuations affect the performance system, such as clear air, haze, rain atmospheric attenuation. In this study, compensation for clear air, haze and rain atmospheric attenuations of eleven-channel DWDM over FSO based on a low pass raised cosine electrical filter (linear equalizer).

The rest of the paper is organized as follows. Section 2 describes our system and methodology. Section 3 evaluates the findings of the experiments. Finally, Section 4 concludes this article.

\section{THE PROPOSED MODEL}

As illustrated in Figure 1, different components of we used components and the parameter of the DWDM system. The eleven-channel DWDM over FSO has separated based on the ITU grid. Briefly, the basic blocks of DWDM-FSO consist of a transmission, medium (FSO-channel), and reception. FSO requires a line of sight configuration between transmitter and receiver. The transmitter side consists of elevenchannel, each channel includes data in (pseudorandom bit sequence generator at $10 \mathrm{Gbps}$ (total capacity is 110 Gbps)), NRZ pulse generator, the laser, and multiplexer, which will multiplex eleven wavelengths. The wavelengths are as follows: $(1561.42 \mathrm{~nm}, 1559.79 \mathrm{~nm}, 1558.17 \mathrm{~nm}, 1558.55 \mathrm{~nm}, 1554.94 \mathrm{~nm}, 1553.33 \mathrm{~nm}$, $1551.72 \mathrm{~nm}, 1550.12 \mathrm{~nm}, 1548.51 \mathrm{~nm}, 1546.92$, and $1545.32 \mathrm{~nm}$ ). The power of the Laser power is $1 \mathrm{dBm}$. The performance of an eleven-channel DWDM system is evaluated in the FSO link under the following atmospheric attenuations: 1) Haze; and 2) rain [23]. The evaluation was performed based on a low pass raised cosine electrical filter (linear equalizer) at the receiver. 


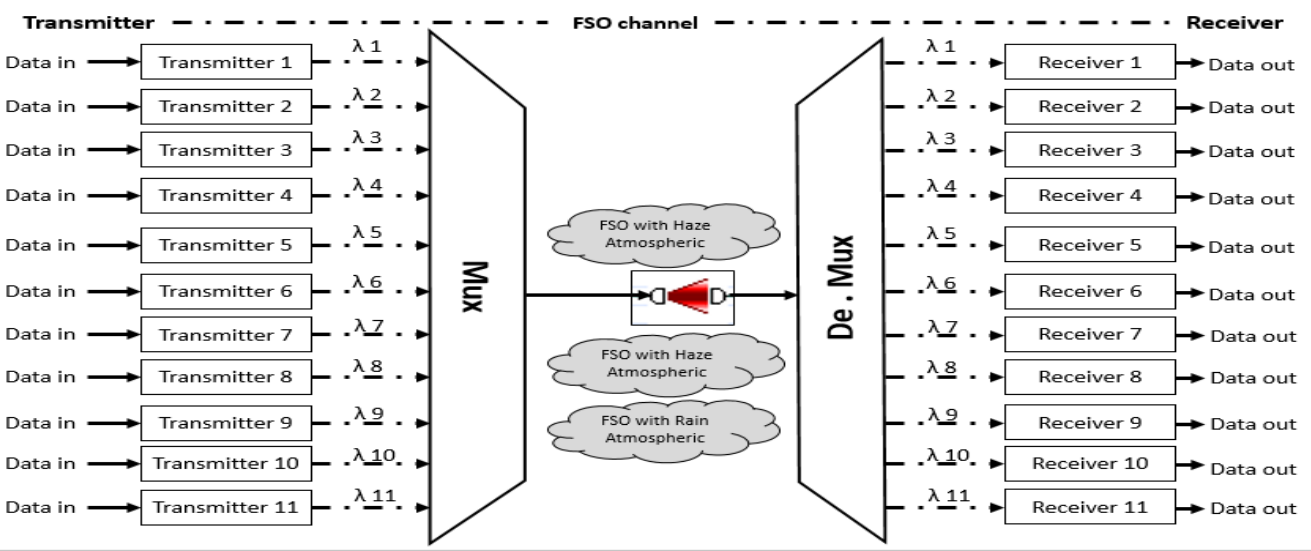

Figure 1. Optical DWDM over FSO under the effect of clear air atmospheric attenuation, haze atmospheric attenuation, and atmospheric rain attenuation

\section{RESULTS AND DISCUSSION}

To achieve a maximum possible medium range of 11-channels, opti-system simulator software has been conducted of DWDM over FSO link under several weather conditions [24]. Table 1 summarizes the medium is based on FSO-channel that transmitted data over atmospheric attenuations. Additionally, based on atmospheric attenuation, the laser power is set to $1 \mathrm{dBm}$, and beam divergence is assigned as $1 \mu \mathrm{rad}$ [25]. As shown in Figure 1, after FSO, the de-multiplexer is made to retrieve the signals from the FSO. The receiver sides are comprised of eleven receivers, and the electrical filter is based on low pass raised cosine.

In the experiments, the performance results of eleven channels DWDM are measured with BER, QFactor and eye diagram over FSO under the effect of clear air, haze, and rain atmospheric attenuations. In the first experiment, Figures 2 and 3 illustrate the BER, Q-Factor results of FSO under the effect of clear air atmospheric attenuation over the distances: 7500 meters, 8000 meters, 8500 meters, 9000 meters, 9500 meters and 10000 meters. Additionally, the eye diagrams of the channels are shown in Figure 4. From the findings of Figures 2-4, it is clear that FSO under the effect of clear air atmospheric attenuation based on DWDM has successfully transmitted data until the distance 9500 meters with acceptable performance for both BER and Q-Factor.

Table 1. Transmitted Data Over Atmospheric Attenuations

\begin{tabular}{cc}
\hline Atmospheric & Attenuation $(\mathrm{dB}-\mathrm{Km})$ \\
\hline Clear air & 0.43 \\
Haze & 4.2 \\
Rain & 5.8 \\
\hline
\end{tabular}

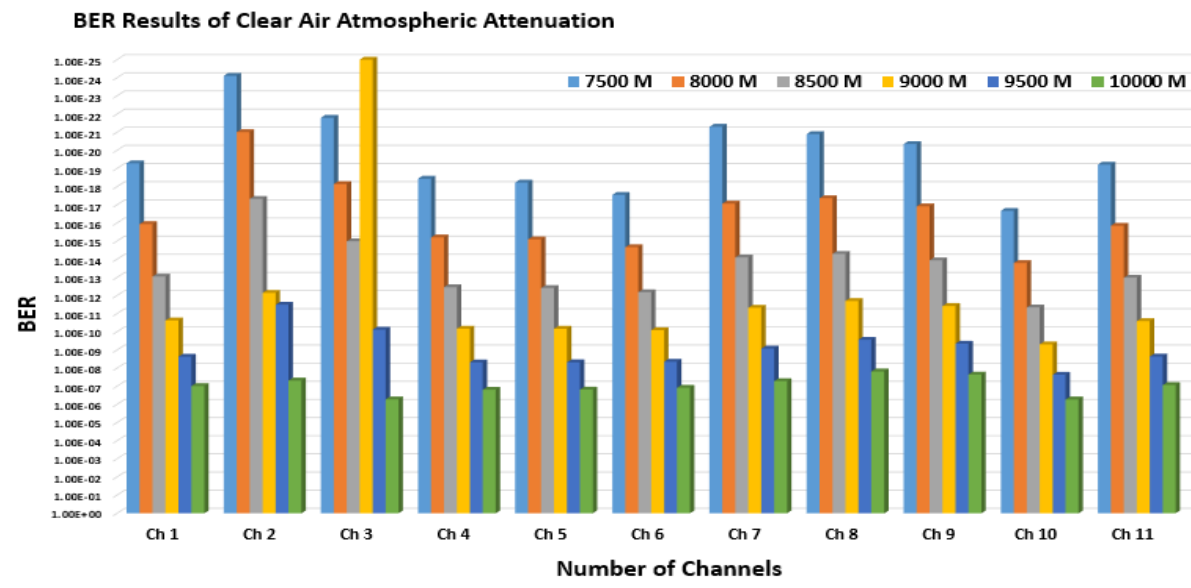

Figure 2. BER results of eleven channels DWDM-FSO under the effect of clear air atmospheric attenuation 


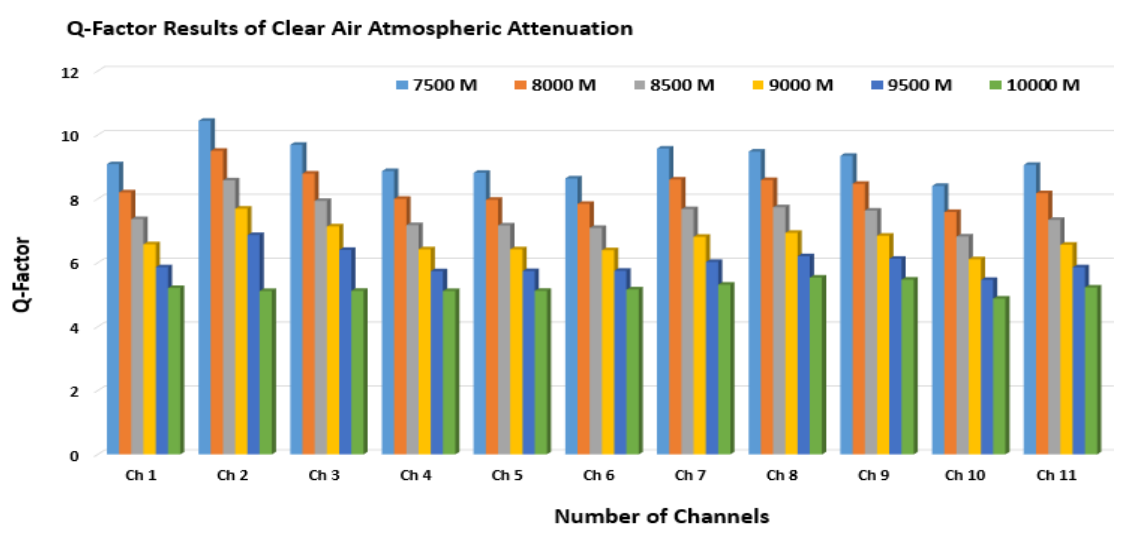

Figure 3. Q-Factor results of eleven channels DWDM-FSO under the effect of clear air atmospheric attenuation

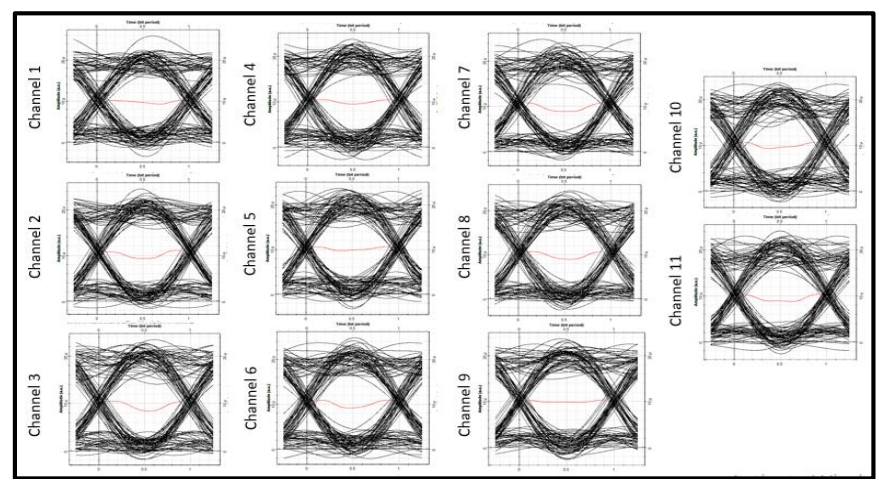

Figure 4. eye diagram results of eleven channels DWDM-FSO under the effect of clear air atmospheric attenuation

In the second experiment, Figures 5 and 6 show the BER, Q-Factor results of FSO under the effect of haze atmospheric attenuation over the distances: 1000 meters, 1500 meters, 2000 meters, 2500 meters, 3000 meters and 3500 meters. Similar to the first simulation, we evaluated the eye diagrams of the channels as shown in Figure 7. According to the outcomes, FSO under the effect of haze atmospheric attenuation based on DWDM has successfully transmitted data until the distance 3000 meters with acceptable performance for both BER and Q-Factor.

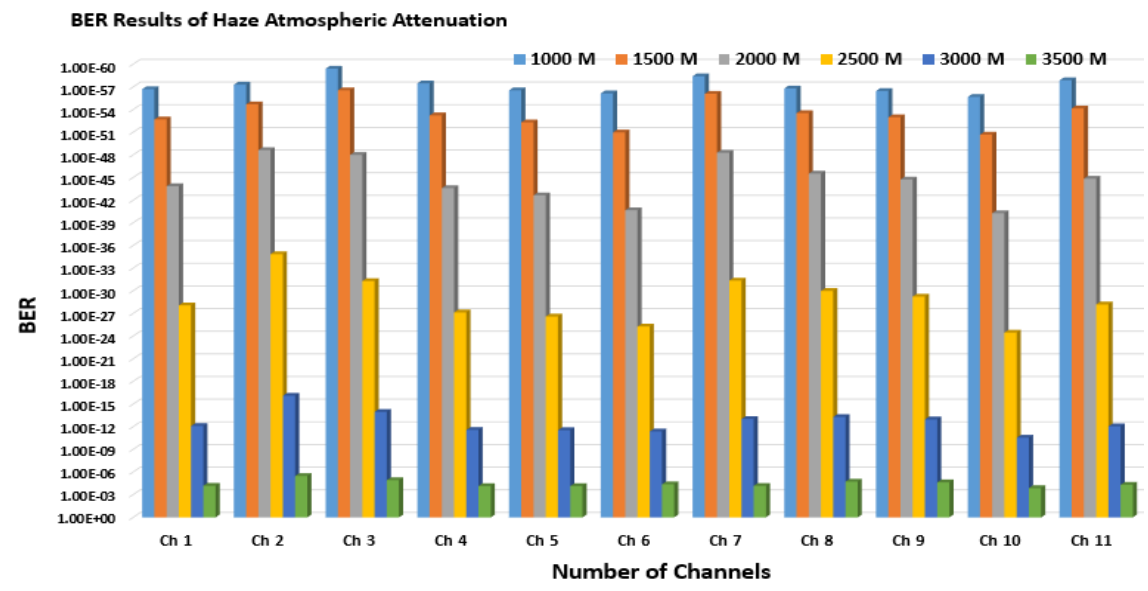

Figure 5. BER results of eleven channels DWDM-FSO under the effect of haze atmospheric attenuation 


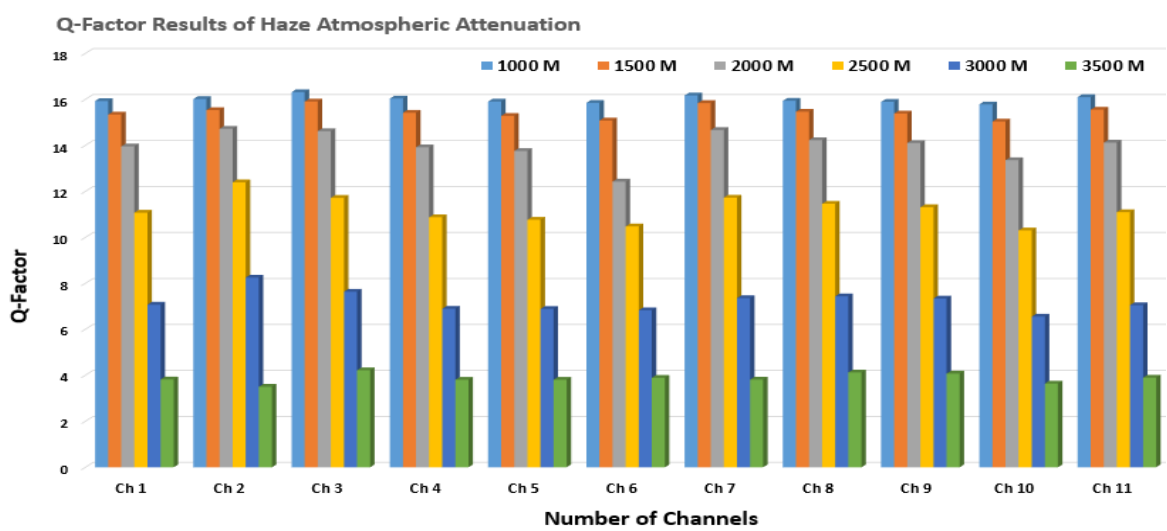

Figure 6. Q-Factor results of eleven channels DWDM-FSO under the effect of haze atmospheric attenuation
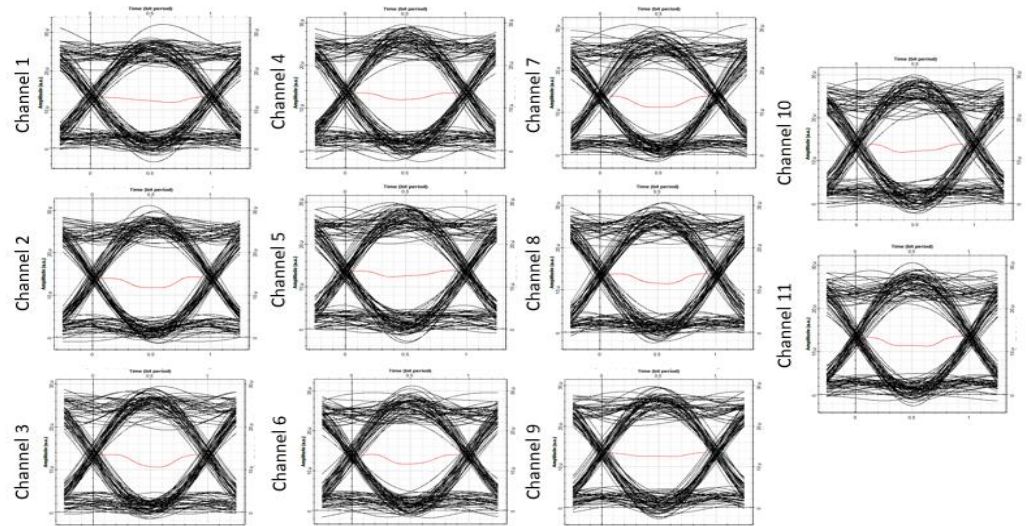

Figure 7. Eye diagram results of eleven channels DWDM-FSO the effect of haze atmospheric attenuation

In the last experiment, Figures 8 and 9 show the BER, Q-Factor results of FSO under the effect of rain atmospheric attenuation over the distances: 500 meters, 1000 meters, 1500 meters, 2500 meters and 300 meters. Additionally, Figure 10 presents the eye diagrams of the channels. The results confirm that FSO under the effect of rain atmospheric attenuation based on DWDM has successfully transmitted data until the distance 2500 meters with a suitable performance for both BER and Q-Factor.

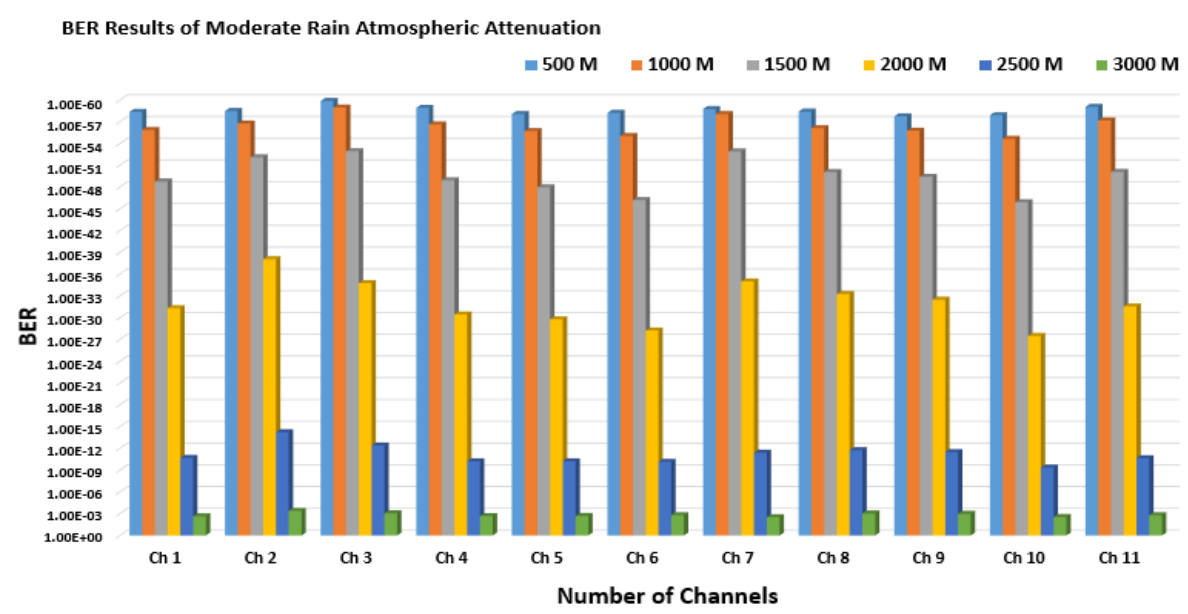

Figure 8. BER results of eleven channels DWDM-FSO under the effect of rain atmospheric attenuation 


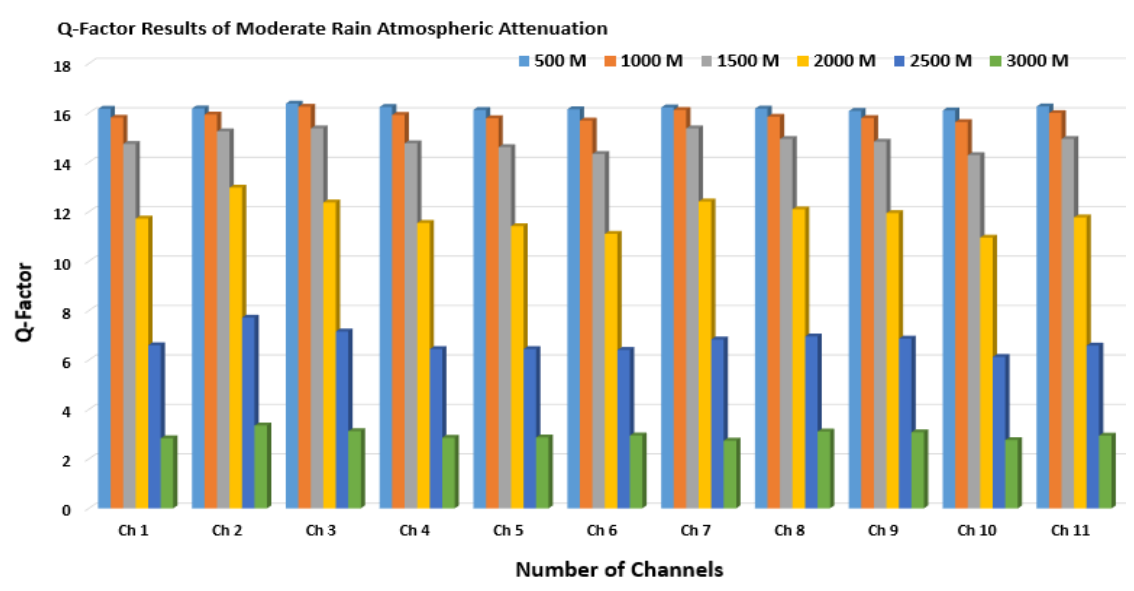

Figure 9. Q-Factor results of eleven channels DWDM-FSO under the effect of rain atmospheric attenuation
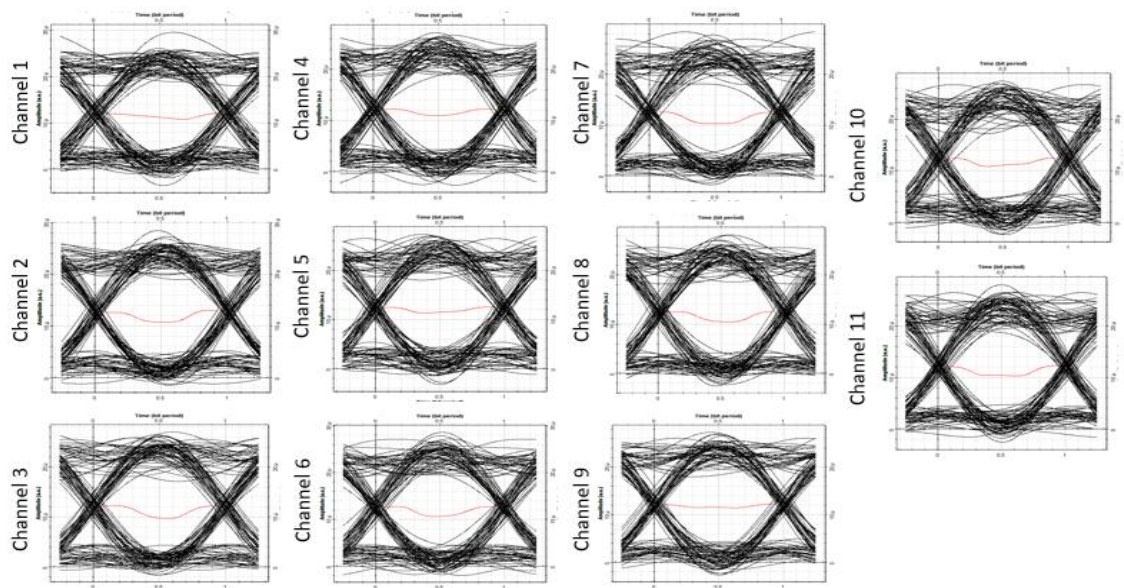

Figure 10. Eye diagram results of eleven channels DWDM-FSO under the effect of rain atmospheric attenuation

\section{CONCLUSION}

In this article, we proposed eleven-channel DWDM-FSO operated over the wavelengths 1561.42 $\mathrm{nm}, 1559.79 \mathrm{~nm}, 1558.17 \mathrm{~nm}, 1558.55 \mathrm{~nm}, 1554.94 \mathrm{~nm}, 1553.33 \mathrm{~nm}, 1551.72 \mathrm{~nm}, 1550.12 \mathrm{~nm}, 1548.51 \mathrm{~nm}$, 1546.92 and $1545.32 \mathrm{~nm}$. In the simulation, $110 \mathrm{Gbps}$ data has been transmitted over eleven-channel under the effect of clear air, haze and rain atmospheric attenuations based on electrical linear equalizer that reached the distances 9500 meters, 300 meters and 2500 meters, respectively. The evaluation was based according to BER, Q-Factor, and eye-diagram.

\section{REFERENCES}

[1] C. V. N. Index, "Forecast and Trends 2017-2022 White Paper," Cisco San Jose, CA, USA, 2019.

[2] V. N. I. Cisco, "Cisco visual networking index: Forecast and trends, 2017-2022," White Pap., vol. 1, 2018.

[3] A. G. Sara Alshwani, Ahmed M. Fakhrudeen, Mohammed Nasih Ismael, Aras Al-Dawoodi, "Hermite-Gaussian Mode in Spatial Division Multiplexing Over FSO System Under Different Weather Condition Based on Linear Gaussian Filter," Int. J. Mech. Eng. Technol., vol. 10, no. 1, pp. 1095-1105, 2019.

[4] M. N. Ismael and A. M. Fakhrudeen, "SDM over hybrid FSO link under different weather conditions and FTTH based on electrical equalization," Int. J. Civ. Eng. Technol., vol. 10, no. 1, pp. 1396-1406, 2019.

[5] A. Noori, A. Amphawan, A. Ghazi, and S. A. A. Ghazi, "Dynamic evolving neural fuzzy inference system equalization scheme in mode division multiplexer for optical fiber transmission," Bull. Electr. Eng. Informatics, vol. 8, no. 1, pp. 127-135, 2019. 
[6] A. Ghazi, S. A. Aljunid, A. Noori, S. Z. S. Idrus, C. B. M. Rashidi, and A. Al-Dawoodi, "Design \& investigation of 10x10 gbit/s MDM over hybrid FSO link under different weather conditions and fiber to the home," Bull. Electr. Eng. Informatics, vol. 8, no. 1, pp. 121-126, 2019.

[7] A. Fareed et al., "Comparison of Different Wavelength Propagations over Few-Mode Fiber based on Space Division Multiplexing in Conjunction with Electrical Equalization," Int. J. Electron. Telecommun., vol. 65, no. 1, pp. 5-10, 2019.

[8] A. Amphawan, A. Ghazi, and A. Al-dawoodi, "Free-space optics mode-wavelength division multiplexing system using LG modes based on decision feedback equalization," in EPJ Web of Conferences, 2017, vol. 162, p. 1009.

[9] M. S. Anuar, S. A. Aljunid, A. R. Arief, and N. M. Saad, "LED spectrum slicing for ZCC SAC-OCDMA coding system," in 7th international symposium on high-capacity optical networks and enabling technologies, 2010, pp. 128-132.

[10] H. Zhang et al., "Performance analysis of FSO system with different modulation schemes over gamma-gamma turbulence channel," in 17th International Conference on Optical Communications and Networks (ICOCN2018), 2019, vol. 11048, p. 1104812.

[11] S. Chaudhary and A. Amphawan, 4 X 2.5Gbps-10 GHz Ro-FSO Transmission Systems by Incorporating Hybrid WDM-MDM of Spiral Phased LG-HG Modes. 2015.

[12] A. Almogahed, A. Amphawan, and Y. Fazea, "Mitigation of Atmospheric Turbulences Using Mode Division Multiplexing based on Decision Feedback Equalizer for Free Space Optics," J. Opt. Commun.

[13] M. A. Khalighi and M. Uysal, "Survey on free space optical communication: A communication theory perspective," IEEE Commun. Surv. tutorials, vol. 16, no. 4, pp. 2231-2258, 2014.

[14] K. Prabu, S. Charanya, M. Jain, and D. Guha, "BER analysis of SS-WDM based FSO system for Vellore weather conditions," Opt. Commun., vol. 403, pp. 73-80, 2017.

[15] M. H. Ali, A. M. Almufti, and H. A. Abu-Alsaad, "Simulative Analyzing of Covering Suburban Areas with $32 \times 10$ Gbps DWDM-PON FTTH Using Different Dispersion and Power,” J. Commun., vol. 14, no. 5, 2019.

[16] P. Krishnan, "Performance Analysis of Hybrid RF/FSO System Using BPSK-SIM and DPSK-SIM Over GammaGamma Turbulence Channel With Pointing Errors for Smart City Applications," IEEE Access, vol. 6, pp. 75025-75032, 2018.

[17] G. Grammel and K. P. Jones, "Wavelength provisioning for customer premise equipment (CPE) in dense wavelength-division multiplex (DWDM) networks." US Patent App. 15/847,385, United States, pp. 1-16, 30 Apr 2019.

[18] S. A. Al-Gailani, M. R. B. Arshad, O. M. Kharraz, and R. Q. Shaddad, "Performance Evaluation of 6-Gbps Hybrid DWDM/Multibeam Free-Space Optical Network in an Unusual Haze," in 10th International Conference on Robotics, Vision, Signal Processing and Power Applications, 2019, pp. 373-380.

[19] M. S. Anuar, S. A. Aljunid, R. Badlishah, N. M. Saad, and I. Andonovic, "Performance analysis of optical zero cross correlation in OCDMA system," J. Appl. Sci., vol. 7, no. 23, pp. 3819-3822, 2007.

[20] S. Parkash, A. Sharma, H. Singh, and H. P. Singh, "Performance investigation of $40 \mathrm{~GB} / \mathrm{s}$ DWDM over free space optical communication system using RZ modulation format," Adv. Opt. Technol., vol. 2016, 2016.

[21] A. Kumar and A. Sharma, "A 5x16 Gbps DWDM system for ground-to-satellite using RZ signaling scheme under different turbulences," Procedia Comput. Sci., vol. 115, pp. 115-122, 2017.

[22] R. Miglani and J. S. Malhotra, "Evaluation of link-compensated 32× 40 Gbit/s DWDM free space optical (FSO) transmission," J. Opt., vol. 47, no. 4, pp. 467-474, 2018.

[23] A. Mansour, R. Mesleh, and M. Abaza, "New challenges in wireless and free space optical communications," Opt. Lasers Eng., vol. 89, pp. 95-108, 2017.

[24] O. Guide, "RSOFT Design Group," Inc, 2010.

[25] A. Amphawan, S. Chaudhary, R. Din, and M. N. Omar, "5Gbps HG 0, 1 and HG 0, 3 optical mode division multiplexing for RoFSO," in Signal Processing \& Its Applications (CSPA), 2015 IEEE 11th International Colloquium on, 2015, pp. 145-149. 